\title{
Cladistic analysis of the subgenus Anopheles (Anopheles) Meigen (Diptera: Culicidae) based on morphological characters
}

\author{
Eliana Collucci, Maria Anice Mureb Sallum ${ }^{+}$ \\ Departamento de Epidemiologia, Faculdade de Saúde Pública, Universidade de São Paulo, Av. Dr. Arnaldo 715, \\ 01246-904 São Paulo, SP, Brasil
}

In the present study, we used morphological characters to estimate phylogenetic relationships among members of the subgenus Anopheles Meigen. Phylogenetic analyses were carried out for 36 species of Anopheles (Anopheles). An. (Stethomyia) kompi Edwards, An. (Lophopodomyia) gilesi (Peryassú), Bironella hollandi Taylor, An. (Nyssorhynchus) oswaldoi (Peryassú) and An. (Cellia) maculatus Theobald were employed as outgroups. One hundred one characters of the external morphology of the adult male, adult female, fourth-instar larva, and pupa were scored and analyzed under the parsimony criterion in PAUP. Phylogenetic relationships among the series and several species informal groups of Anopheles (Anopheles) were hypothesized. The results suggest that Anopheles (Anopheles) is monophyletic. Additionally, most species groups included in the analysis were demonstrated to be monophyletic.

Key words: Culicidae - Anophelinae - Anopheles - morphology - phylogeny

The subfamily Anophelinae consists of three genera: Anopheles Meigen, nearly worldwide in distribution, Bironella Theobald from Australasian region, and the Neotropical Chagasia Cruz (Knight \& Stone 1977, Harbach 2004). Phylogenetic relationships among members of the Anophelinae have been hypothesized based on both morphological and molecular characters (Foley et al. 1998, Sallum et al. 2000, 2002, Krzywinski et al. 2001a, b, Harbach \& Kitching 2005). Unfortunately, results from the studies disagree about the position of Bironella, the monophyly of the subgenus Anopheles and the subgeneric status of Stethomyia Theobald and Lophopodomyia Antunes.

The genus Anopheles consists of 444 formally named and 40 provisionally designated extant species (Harbach 2004, Sallum et al. 2005) subdivided into seven subgenera: Anopheles Meigen, nearly worldwide in distribution, Baimaia Harbach, Rattanarithikul and Harrison (Harbach et al. 2005), restrict to Southeastern Asia, Cellia Theobald, tropical areas of Afrotropical, Oriental, and Australasian regions, and four Neotropical subgenera, Stethomyia, Kerteszia Theobald, Nyssorhynchus Blanchard, and Lophopodomyia. Sallum et al. (2000) synonymized the genus Bironella under Anopheles and considered the subgenera Stethomyia and Lophopodomyia as informal groups within the subgenus Anopheles. In contrast, results of a molecular phylogeny study by Krzywinski et al. (2001a, b) partially corroborated the traditional classification of Anophelinae.

Financial support: Fapesp, grant no. 05/53973-0, CNPq grant no. 472485/2006-7

+Corresponding author: masallum@usp.br

Received 6 October 2006

Accepted 12 March 2007
Harbach and Kitching (2005) mainly based on the morphological data set of Sallum et al. (2000), re-examined the phylogeny of Anophelinae. Consequently, the genus Anopheles s. s. was considered paraphyletic and the clade formed by Bironella, Stethomyia, Lophopodomyia, and Anopheles was treated as "ordinal group", without phylogenetic or subgeneric connotations.

The subgenus Anopheles s. s. consists of 206 nominal species (Harbach 2004). The first infrasubgeneric classification of Anopheles (Anopheles) was proposed by Edwards (1932), who subdivided the subgenus into groups and series. According to Edwards (1932), the Anopheles Group consists of four series, Anopheles (worldwide), Myzorhynchus (Palearctic, Oriental, Australasian and Afrotropical), Cycloleppteron (Neotropical), and Lophoscelomyia (Oriental); and two groups, Arribalzagia (Neotropical) and Christya (Afrotropical). Reid and Knight (1961) adopted Edwards' (1932) classification and consequently subdivided the subgenus Anopheles into two sections, Angusticorn and Laticorn, and six series. The Arribalzagia and Christya Groups of Edwards (1932) were considered series. The Laticorn Section includes the Arribalzagia, Christya, and Myzorhynchus Series, and the Angusticorn Section includes members of the Anopheles, Cycloleppteron, and Lophoscelomyia Series. Members of the former section can be easily recognized by having a funnel-shaped pupal trumpet, whereas those of the latter possess a tubular pupal trumpet. Except for minor changes in the species composition of the groups (Reid 1968, Harrison 1972, Harrison \& Scanlon 1975, Phan et al. 1991, Harbach et al. 2005), the classification within the subgenus Anopheles proposed by Reid and Knight (1961) remains the same.

Classification and phylogenetic relationships among members of the subgenus Anopheles remain unresolved. Foley et al. (1998) and Sallum et al. (2000) found some indication that the subgenus Anopheles may be paraphyletic. In contrast, molecular phylogenetic stud- 
ies of Krzywinski et al. (2001a, b) and Sallum et al. (2002) showed the monophyly of the subgenus Anopheles. However, it is noteworthy that monophyly of the Anopheles may be a result of limited taxon sampling. Except for An. coustani all other Anopheles (Anopheles) species were from the Nearctic and/or Neotropical Regions. Additionally, the Laticorn and Angusticorn Sections were demonstrated to be artificial groups within subgenus Anopheles, whereas the Arribalzagia and Lophoscelomyia Series were monophyletic (Wilkerson \& Peyton 1990, Sallum et al. 2000). Harbach and Kitching (2005) corroborated the polyphyly for the subgenus Anopheles and monophyly of Lophoscelomyia, Arribalzagia, Cycloleppteron, and Myzorhynchus Series.

To assess more objectively the placement of subgenus Anopheles s. s. within Anophelinae it is evident that will be necessary to expand the phylogenetic analyses of Sallum et al. (2000, 2002), Krzywinski et al. (2001a, b), and Harbach and Kitching (2005). The objectives of the present study are: (1) to test the paraphyly of the subgenus Anopheles according to Sallum et al. (2000), and (2) to establish phylogenetic relationships among members of the subgenus.

\section{MATERIALS AND METHODS}

This study includes 38 species of subgenus Anopheles in the ingroup. Unfortunately, no specimen of the Stigmaticus Group of Anopheles (Anopheles) was available for the study. An. gilesi, An. kompi, Bi. hollandi, An. oswaldoi, An. maculatus were employed as outgroups (Table I). The specimens examined are from the Coleção Entomológica da Faculdade de Saúde Pública da Universidade de São Paulo (FSP-USP) and National Museum of Natural History (NMNH), Smithsonian Institution, Washington, D.C.

Taxon sampling was mainly based on both availability of associated males, females, and immature stages and of nominotypical species for each informal group studied (Judd 1996). The hypothesis of phylogenetic relationship among subgenera Cellia and Anopheles (Sallum et al. 2000) and the monophyly of subgenera Nyssorhynchus and Cellia (Wilkerson \& Peyton 1990, Krzywinski et al. 2001a, b, Sallum et al. 2002, Harbach $\&$ Kitching 2005) were utilized for selecting the outgroups (Farris 1982).

Characters listed by Wilkerson and Peyton (1990), Harbach and Kitching (1998, 2005), and Sallum et al. (2000) were either included or reinterpreted. Additionally, we included other characters. Characters obtained from Sallum et al. (2000) data matrix are marked with an asterisk in the character list. All characters were initially treated as unweighted and unordered. Four are parsimony non-informative and were excluded from all analyses, consequently they did not contribute to tree statistics. When more than one character state was observed in a single taxon, these were scored as polymorphic in the data matrix. Missing values were represented by question marks (?) and were employed in the following situations: (1) character-state homology could not be established, (2) character-state assignments were not applicable, and (3) the condition of the available specimens precluded the observation of that character. One hundred-one characters derived from the fourth-instar larvae, pupae, and adult males and females were coded for 41 species: 59 of adults, 30 of fourth instar larvae, and 11 of the pupae. Eighty-six characters were coded as binary and 15 as multistate. Using Sallum's et al. (2000) data set, we selected characters based on phylogenetic information for the subgenus Anopheles and absence of homoplasy. Establishment of primary homologies was based on the criteria proposed by de Pinna (1991) and Nelson (1994). MacClade 4.0 (Maddison \& Maddison 2000) was employed to generate a morphological data matrix (Table II). The character-state homology which could not be established or those which were not applicable for a taxon were scored as (?) and (-), respectively.

The data were analyzed using both equal weights and implied weights implemented by PAUP v4.0b10 (Swofford 2003). The latter applied concavity constant, $K=1$. Characters were considered unordered in all analyses. Character polarization was done a posteriori, using both outgroups and ingroup (Farris 1969, Nixon \& Carpenter 1993). Equally weighted parsimony analyses were performed by heuristic search, using 1000 replications, stepwise addition, random-taxon-addition and Tree Bisection Reconnection (TBR) branch-swapping, and holding one tree per replicate. Four parsimony uninformative characters were excluded from all analyses. Agreement in grouping within a set of equally parsimonious trees was summarized using a strict consensus tree.

Equally weighted parsimony analyses identified a set of 6345 equally parsimonious trees. In the next search round, the characters were weighted using the successive approximation weighting method employing the maximum value of the rescaled consistency index (Farris 1989). This analysis was conducted by heuristic search with 25 random-addition replicates and eight rounds of successive approximation weighting applied to the set of 6345 equally parsimonious trees. Thirty-one characters received weight 1 and 66 characters other than 1 . Character weights stabilized after the third round, identifying three equally parsimonious trees with a weighted length of 7747309 that are not a subset of those 6345 trees. A strict consensus of these three trees is represented in Fig 1. The implied weights analysis, employing the concavity constant, $K=1$, generated three trees. The consensus strict tree is represented in Fig 2.

Clade support was assessed using both bootstrap analysis (Felsenstein 1985) under parsimony criterion and Bremer support (Bremer 1994). Bremer support was estimated using TreeRot v2c (Sorenson 1999), with the same parameters used for the parsimony analysis. Bremer support analysis was carried out using the strict consensus tree of 6345 topologies of the equally weighted analyses with characters assigned equal weights and 20 replications. Bootstrap analysis was carried out using the heuristic search option with 1000 replications, TBR branch-swapping and 10 replicates and one tree hold per replicate. Agreement in grouping within a set of equally parsimonious trees was summarized using a $50 \%$ majority rule consensus tree. Parsimony bootstrap values were plotted on the topology shown in Fig 2. 
Morphological characters

\section{Adult}

1. Erect scales of vertex, development $(\mathrm{CI}=0.500)$ : (0) relatively broad / (1) very narrow.

2. Erect scales of vertex $(\mathrm{CI}=0.333)$ : $(0)$ numerous, present throughout vertex / (1) few, restrict to posterior part of vertex.
All genera of Anophelinae possess erect scales on the vertex. Within the subgenus Anopheles, An. aitkenii James and An. insulaeflorum (Swellengrebel and Swellengrebel de Graaf), both of the Aitkenii Group, exhibit narrow scales on the vertex, whereas in the remaining members of the subgenus Anopheles, the erect scales are broad. In An. implexus (Theobald), An. aitkenii, An. insulaeflorum, and An. algeriensis Theobald

TABLE I

List of Anopheles species used in the morphological cladistic analysis carried out under the parsimony criterion for members of the subgenus Anopheles

\begin{tabular}{|c|c|c|c|c|c|}
\hline Species & Subgenus & Section & Series & Group & Subgroup \\
\hline Bi. hollandi & Brugella & - & - & - & - \\
\hline An. maculatus & Cellia & - & Neocellia & Maculatus & Maculatus \\
\hline An. oswaldoi & Nyssorhynchus & Albimanus & Albimanus & Oswaldoi & Oswaldoi \\
\hline An. gilesi & Lophopodomyia & - & - & - & - \\
\hline An. kompi & Sthetomyia & - & - & - & - \\
\hline An. lindesayi & Anopheles & & Anopheles & Lindesayi & - \\
\hline An. eiseni & Anopheles & & Anopheles & Pseudopunctipennis & - \\
\hline An. algeriensis & Anopheles & Angusticorn & Anopheles & - & - \\
\hline An. aitkenii & Anopheles & Angusticorn & Anopheles & Aitkenii & - \\
\hline An. insulaeflorum & Anopheles & Angusticorn & Anopheles & Aitkenii & - \\
\hline An. sintonioides & Anopheles & Angusticorn & Anopheles & Culiciformis & - \\
\hline An. culiciformis & Anopheles & Angusticorn & Anopheles & Culiciformis & - \\
\hline An. alongensis & Anopheles & Angusticorn & Anopheles & Alongensis & - \\
\hline An. judithae & Anopheles & Angusticorn & Anopheles & Plumbeus & - \\
\hline An. barberi & Anopheles & Angusticorn & Anopheles & Plumbeus & - \\
\hline An. pseudopunctipennis & Anopheles & Angusticorn & Anopheles & Pseudopunctipennis & - \\
\hline An. hectoris & Anopheles & Angusticorn & Anopheles & Pseudopunctipennis & - \\
\hline An. bradleyi & Anopheles & Angusticorn & Anopheles & Punctipennis & - \\
\hline An. crucians & Anopheles & Angusticorn & Anopheles & Punctipennis & - \\
\hline An. punctipennis & Anopheles & Angusticorn & Anopheles & Punctipennis & - \\
\hline An. freeborni & Anopheles & Angusticorn & Anopheles & Maculipennis & Freeborni \\
\hline An. atropos & Anopheles & Angusticorn & Anopheles & Maculipennis & - \\
\hline An. mediopunctatus & Anopheles & Laticorn & Arribalzagia & - & - \\
\hline An. intermedius & Anopheles & Laticorn & Arribalzagia & - & - \\
\hline An. implexus & Anopheles & Laticorn & Christya & - & - \\
\hline An. annulipalpis & Anopheles & Angusticorn & Cycloleppteron & - & - \\
\hline An. grabhamii & Anopheles & Angusticorn & Cycloleppteron & - & - \\
\hline An. asiaticus & Anopheles & Angusticorn & Lophoscelomyia & Asiaticus & Asiaticus \\
\hline An. interruptus & Anopheles & Angusticorn & Lophoscelomyia & Asiaticus & Interruptus \\
\hline An. hyrcanus & Anopheles & Laticorn & Myzorhynchus & Hyrcanus & - \\
\hline An. sinensis & Anopheles & Laticorn & Myzorhynchus & Hyrcanus & - \\
\hline An. paraliae & Anopheles & Laticorn & Myzorhynchus & Hyrcanus & Lesteri \\
\hline An. nigerrimus & Anopheles & Laticorn & Myzorhynchus & Hyrcanus & Nigerrimus \\
\hline An. coustani & Anopheles & Laticorn & Myzorhynchus & Coustani & - \\
\hline An. bancroftii & Anopheles & Laticorn & Myzorhynchus & Bancroftii & - \\
\hline An. pseudobarbirostris & Anopheles & Laticorn & Myzorhynchus & Bancroftii & - \\
\hline An. barbirostris & Anopheles & Laticorn & Myzorhynchus & Barbirostris & Barbirostris \\
\hline An. pollicaris & Anopheles & Laticorn & Myzorhynchus & Barbirostris & Barbirostris \\
\hline An. baezai & Anopheles & Laticorn & Myzorhynchus & Umbrosus & Baezai \\
\hline An. letifer & Anopheles & Laticorn & Myzorhynchus & Umbrosus & Letifer \\
\hline An. umbrosus & Anopheles & Laticorn & Myzorhynchus & Umbrosus & Umbrosus \\
\hline An. albotaeniatus & Anopheles & Laticorn & Myzorhynchus & Albotaeniatus & - \\
\hline An. montanus & Anopheles & Laticorn & Myzorhynchus & Albotaeniatus & - \\
\hline
\end{tabular}




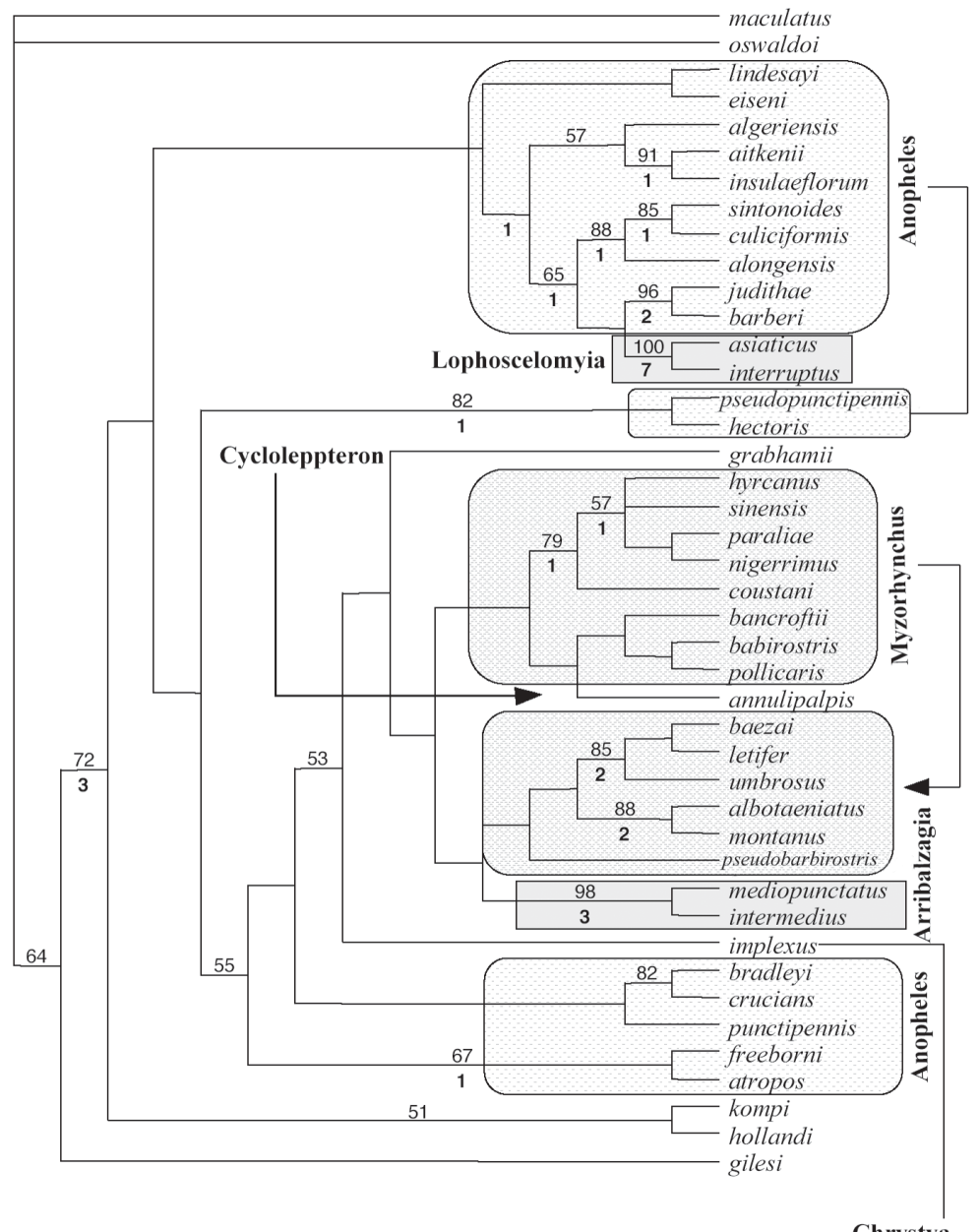

Chrystya

Fig. 1: strict consensus tree of three most parsimonious topologies generated in the fourth round of the successive weightings analysis, using morphological data for 43 taxa of Anophelinae and 101 unordered characters. Bootstrap support values are indicated above tree branches, while Bremer support values are in bold below the branches. Series of the subgenus Anopheles are indicated in the topology.

the erect scales are restricted to posterior part of vertex. This condition also occurs in An. kyondawensis Abraham, An. corethroides Theobald and in members of Stethomyia and Bironella (Sallum et al. 2000, Harbach \& Kitching 2005).

3. Decumbent scales on vertex $(C I=0.333)$ : $(0)$ absent / (1) present.

4. Lateral scales on clypeus $(C I=1.000)$ : $(0)$ absent / (1) present.

Among the species included in the present study, only An. paraliae Sandosham, An. nigerrimus Giles, An. hyrcanus (Pallas), An. sinensis Wiedemann, and An. coustani Laveran possess lateral scales on the clypeus. Presence of lateral scales on clypeus is a non-homoplastic synapomorphy that supports the sister group relationship between Hyrcanus and Coustani Groups.

5 . Lateral lobes of gena $(\mathrm{CI}=0.333)$ : $(0)$ not protuberant / (1) protuberant.

6*. Scales on pedicel $(\mathrm{CI}=0.250)$ : (0) present / (1) absent.
7. Labelum ( $\mathrm{CI}=0.500)$ : $(0)$ comprising one sclerite / (1) two incompletely separated sclerites.

In An. corethorides and An. kyondawensis the labelum consists of one sclerite (Harbach \& Kitching 2005).

8. Scales on antennal flagellomere $2(\mathrm{CI}=0.500)$ : (0) present / (1) absent.

9.* Maxillary palpus, palpomere $2(\mathrm{CI}=0.250)$ : $(0)$ with erect scales / (1) with decumbent scales.

10. * Female palpomere 2, dorsal surface $(\mathrm{CI}=$ 1.000): (0) without pale scales / (1) with pale scales.

11. Scales on anterior promontory $(\mathrm{CI}=1.000)$ : $(0)$ absent / (1) present.

12. Scales on dorsal area of antepronotum $(\mathrm{CI}=$ 0.250): (0) present / (1) absent.

13. Scutum, profile in dorsal view $(\mathrm{CI}=1.000)$ : (0) elongate / (1) short.

14. Scales on ventral area of antepronotum $(\mathrm{CI}=$ 0.250): (0) present / (1) absent.

Whereas An. asiaticus Leicester, An. interruptus Puri, 


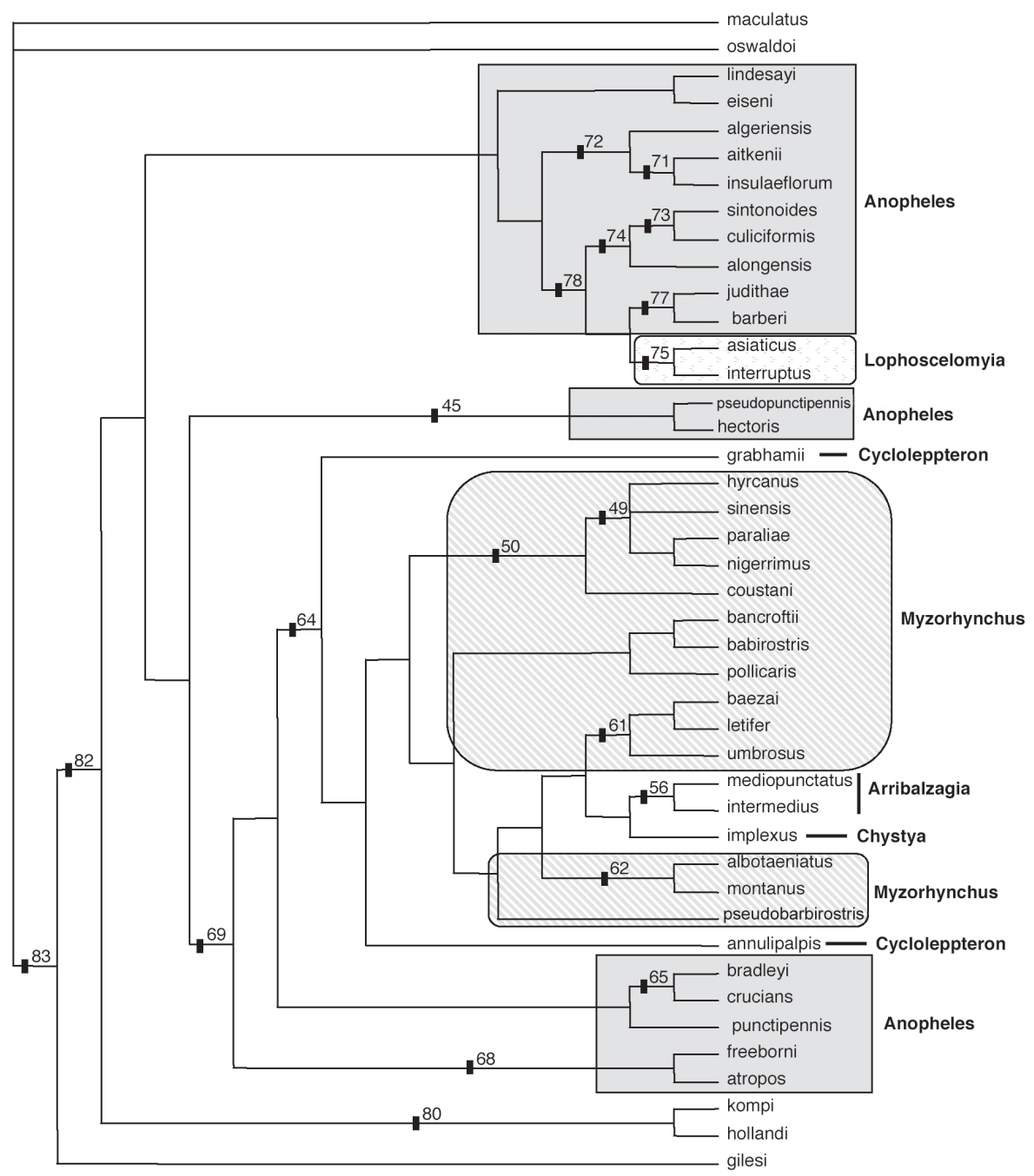

Fig. 2: strict consensus tree of three topologies generated under the implied weights analysis, using the concavity index $K=1$. Implied weights analysis employed a morphological data for 43 taxa of Anophelinae and 101 unordered characters. The rectangles on the branches and the numbers on the right side indicate the nodes for which characters were optimized, as shown in Table III.

An. mediopunctatus (Lutz), An. intermedius (Peryassú), An. implexus, and An. pollicaris Reid have scales on the ventral part of the antepronotum, An. barbirostris Van der Wulp and An. oswaldoi (Peryassú) show both conditions.

15.* Upper proepisternal scales $(\mathrm{CI}=0.250)$ : $(0)$ present / (1) absent.

16. Anterior mesepimeral scales $(\mathrm{CI}=1.000)$ : $(0)$ present / (1) absent.

Among all the species included in this study, only An. pollicaris, An. barbirostris, An. annulipalpis Lynch Arribalzaga, and An. bancroftii Giles possess anterior mesepimeral scales.

17.* Lower mesepimeral setae $(\mathrm{CI}=0.250)$ : $(0)$ present / (1) absent.

18.* Prescutellar scales $(\mathrm{CI}=1.000)$ : $(0)$ present / (1) absent.

The presence of scales on prescutellar area is a synapomorphy for An. judithae Zavortink and An. barberi Coquillett.
19. Supraalar scales $(\mathrm{CI}=0.250)$ : (0) present / (1) absent.

20. Scales on posterolateral surface of the hindcoxa at the edge of the articulation with trochanter $(\mathrm{CI}=$ 0.250): (0) absent / (1) present.

Harbach and Kitching (2005) considered these scales to be situated on the anterodistal surface of the hindcoxa.

21.* Hindtibia $(\mathrm{CI}=0.286)$ : (0) mostly dark-scaled with band of pale scales / (1) mostly dark-scaled with sparse pale scales / (2) entirely dark-scaled.

22. Apical tuft of sub-erect scales on hindfemur (CI = 1.000): (0) absent / (1) present.

An. interruptus and An. asiaticus possess a tuft of sub-erect scales at apex of hindfemur. This condition also occurs in An. (Lophopodomyia) squamifemur Antunes.

23. Hindtarsomere $5(\mathrm{CI}=0.500)$ : $(0)$ mostly or entirely dark-scaled / (1) pale-scaled.

24.* Posterolateral tuft of sub-erect scales on abdominal tergum IV (CI = 0.333): (0) present / (1) absent.

An. oswaldoi, An. implexus, An. intermedius, and An. 
mediopunctatus exhibit tufts of semi-erect scales arising posterolaterally on abdominal tergum IV. The presence of the tuft of lateral scales on abdominal terga is also observed in other species of the Arribalzagia Series.

25. Sternum VII (female) $(C I=0.333)$ : $(0)$ without scales / (1) with scales.

26.* Costal vein $(\mathrm{CI}=0.333)$ : $(0)$ with spots of pale and dark scales / (1) entirely covered with dark scales.

27. Subcostal dark (SCD) spot $(C I=1.000)$ : $(0)$ absent / (1) present.

Species included in the Arribalzagia Series possess subcosta vein ending in a conspicuous, isolated dark spot distal to the sector dark spot. According to Wilkerson and Peyton (1990) the presence of SCD spot on subcostal area (SCA) is an apomorphic condition for the Arribalzagia Series.

28. Accessory sector pale (ASP) spot $(\mathrm{CI}=0.333)$ : (0) present / (1) absent.

The ASP spot is absent in species of the subgenus Anopheles, except in An. pseudopunctipennis Theobald complex and An. intermedius.

29. Vein $\mathrm{R}_{2+3}$ scales, dorsal surface $(\mathrm{CI}=0.250)$ : (0) linear / (1) spatulate.

30. Humeral crossvein, dorsal surface $(\mathrm{CI}=0.333)$ : (0) without scales / (1) with scales.

\section{Male genitalia}

31. Epandrium (tergum IX) (CI = 1.000): (0) mainly membranous / (1) moderately sclerotized / (2) largely sclerotized.

32. Ninth tergal lobes $(\mathrm{CI}=0.333)$ : (0) present / (1) absent.

33. Ninth tergal lobes $(C I=1.000)$ : $(0)$ weakly developed / (1) strongly developed.

Harrison and Scanlon (1975) suggested that the degree of development of the lobes of tergum IX is important to identify species of the subgenus Anopheles. Among species of this subgenus, An. aitkenii, An. insulaeflorum, An. algeriensis, An. sintonoides Ho and An. culiciformis Cogill, An. alongensis Venhuis show no evidence of ninth tergal lobe. In An. pseudopunctipennis Baisas and An. hectoris Giaquinto-Mira, the epandrium consists of a narrow sclerotized plate with poorly developed lobes. In the remaining species of the subgenus Anopheles included in this study, the epandrium has well developed lobes.

34. Dorsal claspette (parsimony uninformative): (0) poorly developed / (1) strongly developed.

35. Dorsal claspette $(\mathrm{CI}=0.667)$ : $(0)$ well separate from ventral claspette / (1) poorly separate from ventral claspette (2) fused on ventral claspette.

The dorsal claspette is poorly developed in An. gilesi Peryassu. In Nyssorhynchus it is well developed and separate from the ventral claspette, whereas in the subgenera Cellia and Anopheles the dorsal claspette is short and very close to the ventral claspette. An. bradleyi King, An. punctipennis (Say) and An. crucians Wiedemann possess dorsal claspette and ventral claspette fused, forming a unique paired lobe.

36.* Setae of dorsal claspette $(\mathrm{CI}=0.200)$ : (0) broad / (1) narrow.
37.* Setae of dorsal claspette $(C I=0.400)$ : (0) separate, spine-like / (1) separate, flattened / (2) partially fused, forming a club-like on inner half but separate on outer half / (3) partially fused, forming a distal club, with separate basal stems / (4) fused, forming a complete club.

38. $*$ Seta of dorsal claspette $(\mathrm{CI}=0.667)$ : (0) comprising a single group / (1) two groups / (2) absent.

In An. sintonoides, An. culiciformis, An. asiaticus, and An. alongensis the setae of dorsal claspette is divided in two distinct groups. The specialized seta of dorsal claspette are absent in Bironella hollandi Taylor and An. gilesi.

39.* Ventral claspette, development (parsimony uninformative): (0) a single ventromedial lobe / (1) two somewhat mound-like lobes / (2) two columnar, well separated lateral lobes / (3) four columnar lobes / (4) two columnar lobes, with an enlarged membranous distally expanded appendage.

40. Setae of ventral claspette $(C I=0.500)$ : $(0)$ absent / (1) present.

In members of the Nyssorhynchus, the ventral claspette occurs as a single median lobe, placed ventrally between the gonocoxites, whereas in the majority of the species of subgenera Anopheles and Cellia the ventral claspette is a short, mound-like lobe. In An. mediopunctatus the ventral claspette is large and columnar, whereas An. kompi Edwards possesses the ventral claspette developed in four columnar lobes and $B i$. hollandi exhibits condition (4).

41. Parabasal spines $(\mathrm{CI}=1.000)$ : (0) present / (1) absent.

$42 *$. Number of parabasal spines $(\mathrm{CI}=0.500)$ : $(0)$ one / (1) two / (3) four.

Christophers (1915) introduced the term "parabasal spines" to define a pair of specialized setae that arises from a lobe at the base of the dorsomesal surface of the gonocoxite. Subsequently, the parabasal spines have been used for subgeneric classification of the genus Anopheles without the establishment of homologies among members of Anopheles (Harbach \& Kitching 2005).

Within the subgenera Kerteszia and Nyssorhynchus, a single differentiated parabasal spine arises from a welldeveloped tubercle placed basal on tergomesal margin of gonocoxite, whereas in the subgenus Cellia four or five parabasal spines are present on basal half of dorsolateral surface. Within the subgenus Anopheles the number of parabasal spines varies from one spine to three spines, normally arising from a small protuberance located on basal half of dorsolateral surface. In our analyses, we scored parabasal spines as either absent or undifferentiated in Bi. hollandi and An. kompi, while An. gilesi possesses one parabasal spine arising from dorsomesal margin, displaced from base.

43. * Gonocoxite, internal spine $(\mathrm{CI}=0.500):(0)$ absent / (1) present.

44. * Internal spine, placement $(\mathrm{CI}=1.000):(0)$ near apex of the gonocoxite / (1) near middle length of the gonocoxite.

The internal spine is absent in members of Cellia and Bironella. In An. aitkenii, An. insulaeflorum, and An. algeriensis the internal spine arises near apex of the gonocoxite.

45. Gonocoxite, accessory spines $(\mathrm{CI}=0.333)$ : (0) 


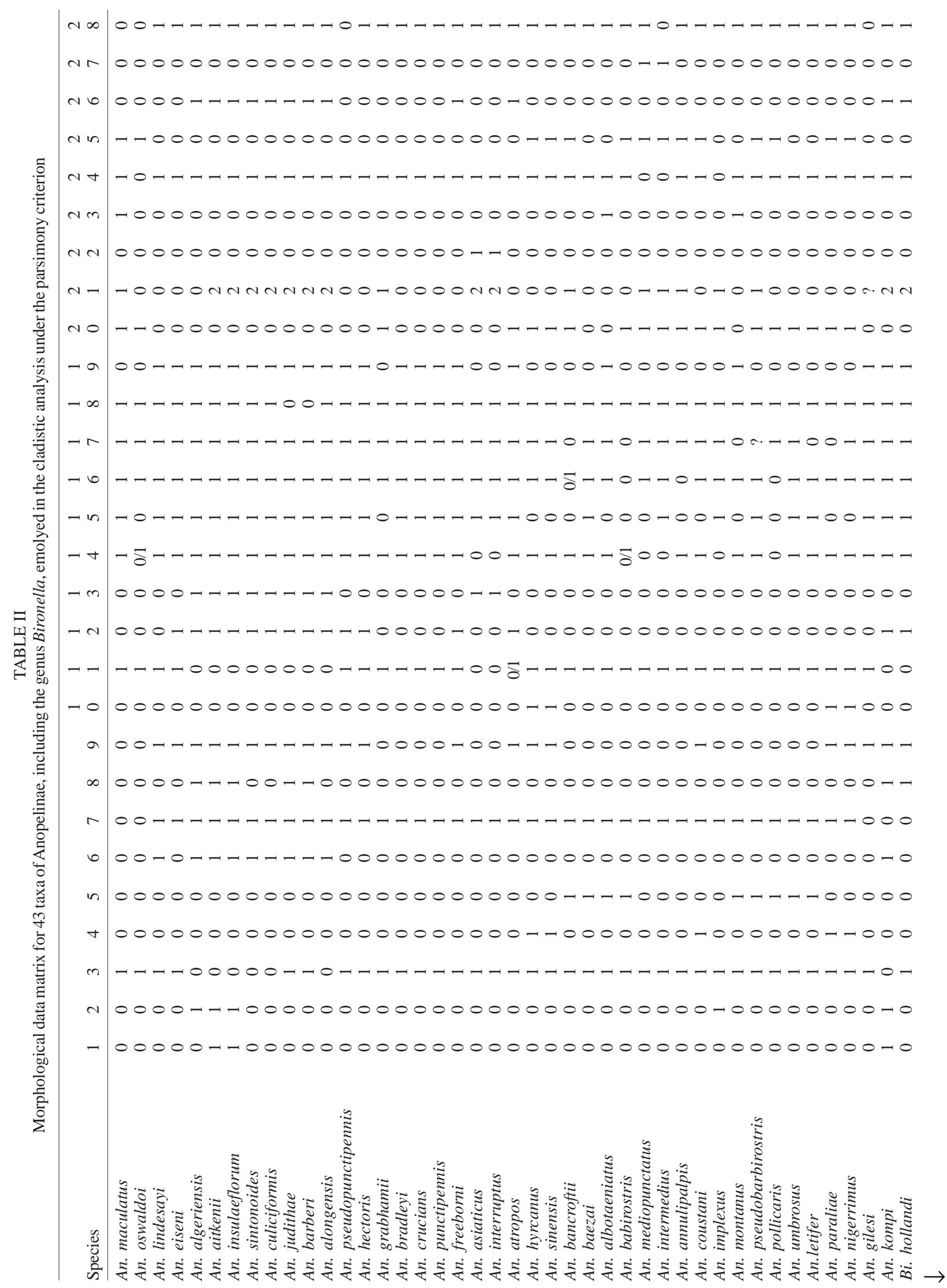




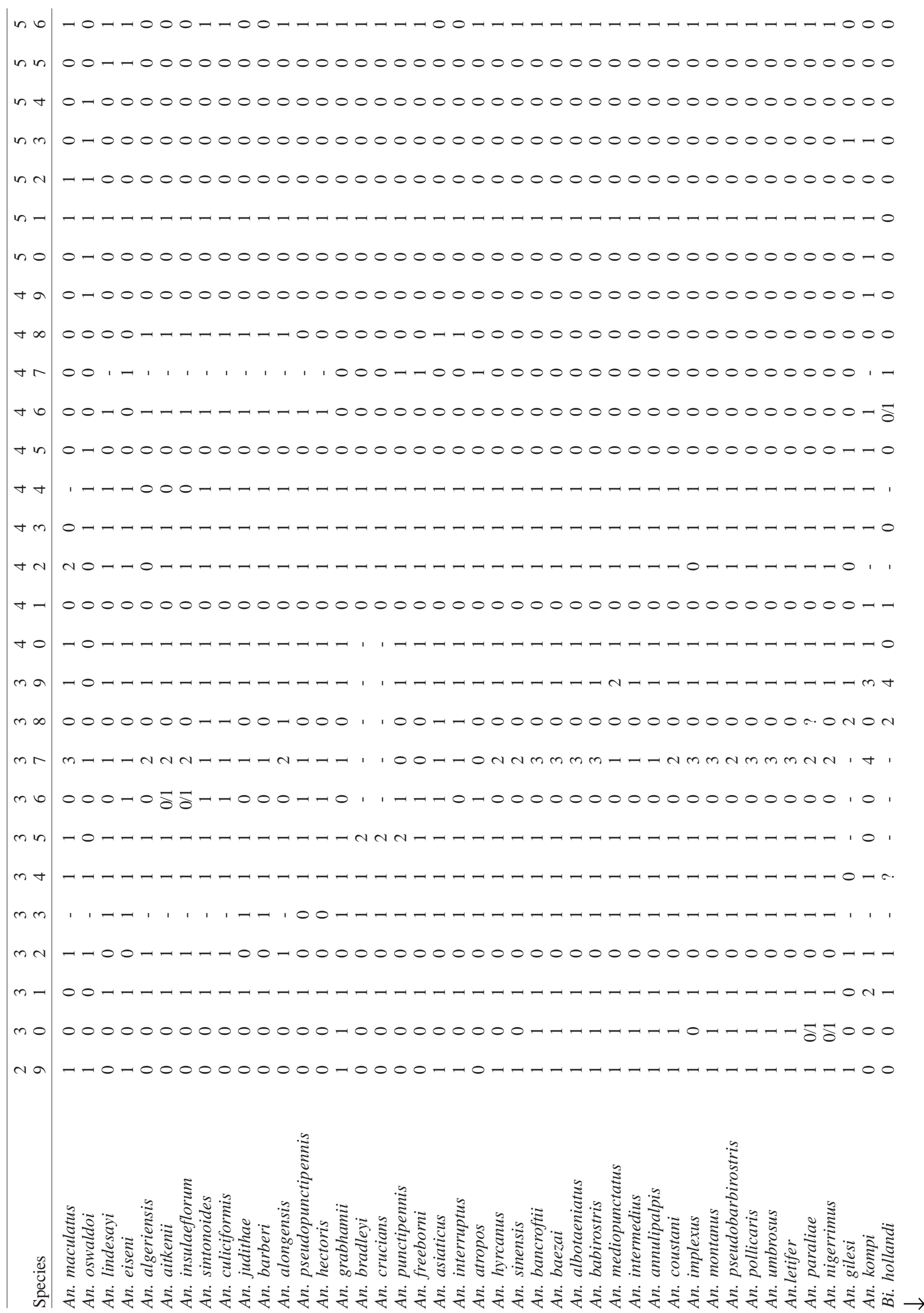




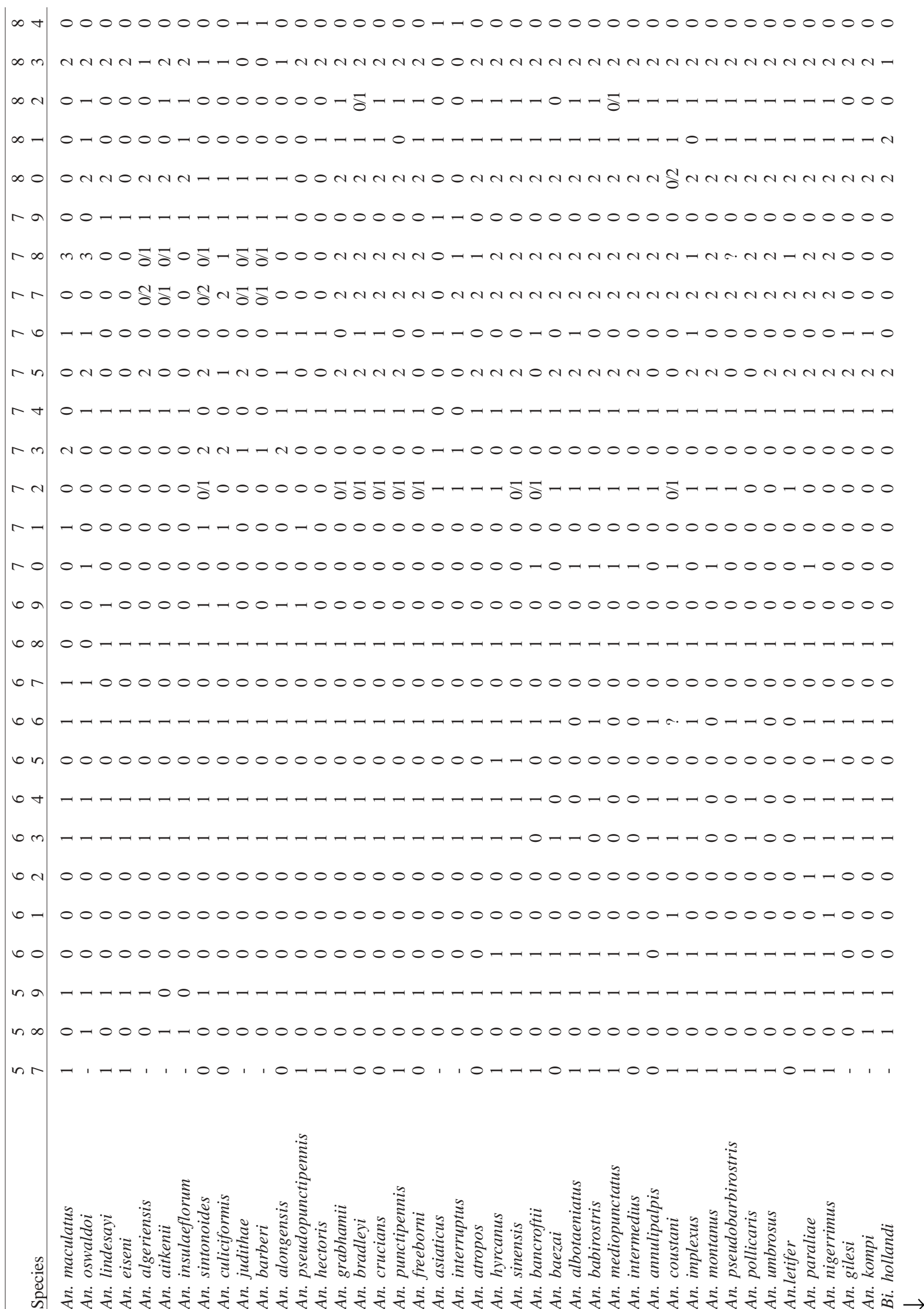




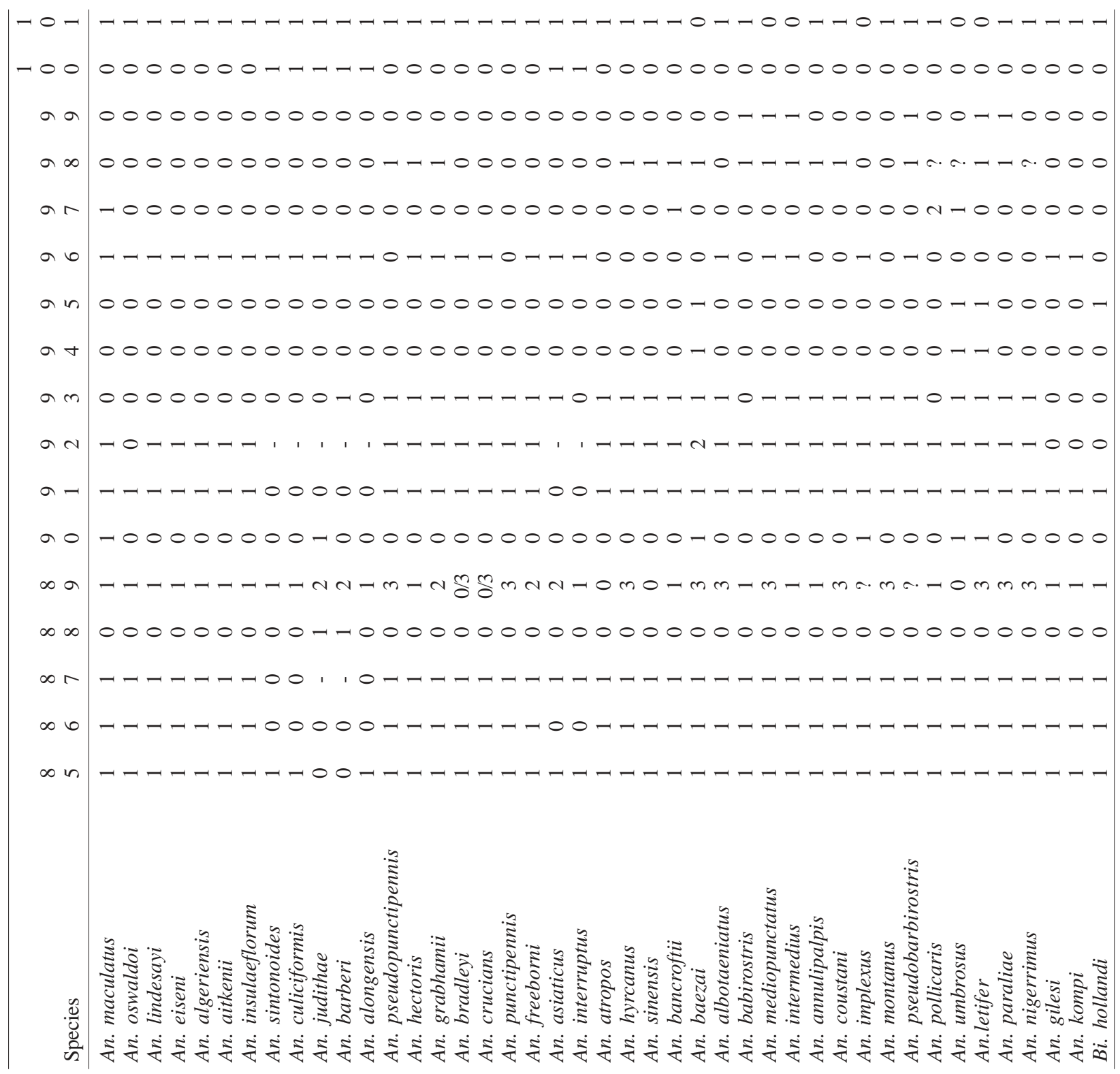

absent / (1) present

According to Sallum et al. (2000), the accessory spines are two long, strong, apically flattened, hooked spines that arise from two adjacent, prominent tubercles on the tergomesal surface of the gonocoxite. This condition occurs in Nyssorhynchus and Kerteszia species. In the study, we scored a single, strong, mid-dorsal spine inserted on the dorsomesal surface of the gonocoxite as homologous to the accessory spine of Kerteszia and Nyssorhynchus. Additionally, An. gilesi possess two accessory spines. We scored the accessory spines as either absent or undifferentiated in Bironella, Cellia, and Anopheles

46. * Scales on lateral surface of the gonocoxite (CI $=0.250)$ : (0) present / (1) absent.

47. Scales on lateral surface of the gonocoxite $(\mathrm{CI}=$ 0.250): (0) extending from base toward apex of lateral and dorsal surfaces / (1) restricted to basolateral surface.

In An. alongensis, An. lindesayi Giles, An. algeriensis, An. aitkenii, An. insulaeflorum, An. judithae, An. barberi, An. culiciformis, and An. sintonoides, the gonocoxite does not possess scales on lateral and dorsal surfaces, whereas Bi. hollandi, An. eiseni Coquillett, An. bradleyi, An. atropos Dyar and Knab, An. freeborni Aitken, and An. punctipennis exhibit few scales restricted to basolateral surface of the gonocoxite.

48.* Gonocoxite, shape $(\mathrm{CI}=1.000)$ : $(0)$ conical / (1) columnar.

Condition 1 is a synapomorphy for An. judithae, An. barberi, An. asiaticus, An. interruptus, An. alongensis, An. culiciformis, An. sintonoides, An. algeriensis, An. insulaeflorum, and An. aitkenii.

49. Apodeme of gonocoxite $(\mathrm{CI}=0.500)$ : $(0)$ narrow / (1) broad. 
50. Dorsomedial rim of gonocoxite $(\mathrm{CI}=0.500)$ : (0) short / (1) long.

51.* Tergomedial seta of gonocoxite (parsimony uninformative): (0) absent / (1) present.

52. Apicomedial setae of gonocoxite $(\mathrm{CI}=1.000)$ : (0) absent / (1) present.

The apicomedial seta occurs in members of Cellia and Nyssorhynchus.

53.* Gonostylus claw (CI = 0.333): (0) short / (1) long.

54.* Apex of gonostylus claw (parsimony uninformative): (0) blunt / (1) acute.

55.* Gonostylus claw, placement $(\mathrm{CI}=1.000):(0)$ arising subapically / (1) arising apically.

Similarly to An. oswaldoi and An. maculatus Theobald, gonostylus claw arises subapically in the majority of the species of subgenus Anopheles employed in the study. In An. kompi, An. lindesayi and An. eiseni gonostylus claw is inserted apically.

56. Aedeagal leaflets (CI = 0.333): (0) absent / (1) present.

57.* Margin of distal aedegal leaflets $(\mathrm{CI}=0.143)$ : (0) smooth / (1) with serrations or denticles on one or both edges.

58. Process of sternum $X(C I=0.333)$ : (0) poorly sclerotized / (1) well sclerotized.

59. Process of sternum $X(C I=1.000)$ : (0) with dorsal and ventral extensions / (1) without dorsal and ventral extensions.

\section{Pupa}

60. Pupal trumpet $(\mathrm{CI}=0.333)$ : (0) angusticorn / (1) laticorn.

Condition (1) is apomorphic for species of Laticorn Section and occurs independently in An. darlingi Root, Bironella, and Chagasia.

61.* Pupal trumpet $(\mathrm{CI}=0.500)$ : $(0)$ with parallel vertical wrinkles / (1) without parallel vertical wrinkles.

Condition (0) occurs in An. coustani and in species of the Hyrcanus Group.

62.* Pina $(\mathrm{CI}=1.000)$ : (0) smooth along edge / (1) denticulate along edge.

63. Secondary meatal cleaft $(C I=0.250)$ : $(0)$ present / (1) absent.

64. Tragus ( $\mathrm{CI}=0.500)$ : (0) present / (1) absent.

The presence of a secondary meatal cleft occurs in several species of the Myzorhynchus and Arribalzagia Series and occurs independently in Bi. gracilis Theobald. The presence of the tragus was observed in An. albotaeniatus (Theobald), An. montanus Stanton and Hacker, An. umbrosus (Theobald), An. letifer Sandosham, An. baezai Gater of the Myzorhynchus Series and in An. mediopunctatus and An. intermedius of the Arribalzagia Series.

65.* Palpal case $(\mathrm{CI}=0.500)$ : $(0)$ similar in development in the pupa male and female / (1) longer and slender in the pupa male than in the pupa female.

Members of the Hyrcanus Group exhibit sexual dimorphism for this character. In An. hyrcanus, An. nigerrimus, and An. sinensis, the length of the palpal case of the male pupa is twice that of the female. In the remaining species included in the present study both pu- pae male and female show palpal case similar in length and development.

66. Denticles on posterior margin of the abdominal terga II-VII (CI = 0.333): (0) present / (1) absent.

In An. mediopunctatus, An. intermedius, An. albotaeniatus, An. letifer, and An. umbrosus the posterior margin of the abdominal terga II-VII possess small denticles, which are also present in other members of the Umbrosus Group and Chagasia.

67.* Seta 1-V (CI = 1.000): (0) short / (1) very long.

In An. oswaldoi and An. maculatus, seta $1-\mathrm{V}$ is very long, extending beyond the following segment.

68.* Seta 1-V (CI = 1.000): (0) single / (1) branched.

69.* Seta 5-V (CI = 0.333): (0) developed, shorter than segment V / (1) well developed, as long as segment V.

70. Posterolateral lobe of the abdominal segment VIII (CI = 0.167): (0) absent / (1) present.

71. * Seta 1-P (CI = 0.333): (0) straight / (1) curved at apex.

Condition (1) is present in An. maculatus, An. pseudopunctipennis, An. culiciformis and An. sintonoides.

72. Seta 1-P (CI = 0.200): (0) single / (1) branched.

\section{Larva}

73. Seta 1-A (CI = 0.667): (0) strongly developed, longer than width of antenna at the point of insertion / (1) moderately developed, shorter than width of antenna at the point of insertion / (2) minute.

74. * Seta 1-A (CI = 0.333): (0) single / (1) branched.

75. Seta $2-C$, placement $(C I=0.167)$ : $(0)$ anterior to seta 3-C / (1) posterior to 3-C / (2) directly mesal to 3-C.

76. Setae $2-C(C I=0.111)$ : $(0)$ inserted very close to each other, distance between insertions of both 2-C is smaller than the width of basal alveolus / (1) inserted more separate, distance between insertions of both 2-C is greater than the width of basal alveolus.

77. * Seta 3-C, development $(\mathrm{CI}=0.333)$ : (0) single / (1) bifid / (2) branched.

78. Seta 3-C (CI = 0.429): (0) simple / (1) with few secondary apical branches / (2) with several apical branches (dendritic) / (3) aciculate.

79.* Seta 3-C (CI = 0.500): (0) long, 0.7 length of 2-C / (1) short, 0.5 length of 2-C.

80.* Seta 4-C, placement $(\mathrm{CI}=0.400)$ : (0) arising mesal to seta 3-C / (1) lateral to seta 3-C.

81. Seta $4-C$, development $(C I=0.222)$ : (0) strongly developed / (1) poorly developed / (2) minute.

82.* Seta 4-C (CI = 0.250): (0) single / (1) branched.

83. * Seta 5-C (CI = 0.500): (0) simple / (1) aciculate / (2) plumose.

84. * Seta 6-C, development $(\mathrm{CI}=0.500)$ : $(0)$ as long as 5-C / (1) shorter than 5-C.

85.* Seta 7-C (CI = 1.000): (0) single / (1) branched.

86. Primary branch (rachis) of seta 7-C $(\mathrm{CI}=1.000)$ :

(0) short, sub equal in length to 4-C / (1) longer than 4-C.

87. Secondary branch of seta $7-\mathrm{C}(\mathrm{CI}=1.000)$ : $(0)$ short / (1) long.

88.* Seta 0-P (CI = 1.000): (0) present / (1) absent.

The character 87 was first proposed by Zavortink (1969). Condition (1) is present in An. judithae and An. barberi and probably represents a synapomophy for the Plumbeus Group. 
89.* Seta 1-P (CI = 0.273): (0) simple / (1) branched, filamentous / (2) branched, aciculate / (3) with apical branches.

90. Secondary branch of seta $3-\mathrm{T}(\mathrm{CI}=0.250)$ : $(0)$ palmate / (1) not palmate.

91. * Seta 1-I (CI = 1.000): (0) single / (1) branched.

92. Secondary branch of seta $1-\mathrm{I}(\mathrm{CI}=0.500)$ : $(0)$ broad / (1) narrow / (2) very narrow.

93. Seta 1-II (CI = 0.167): (0) palmate / (1) not palmate.

94. Seta 1-VII (CI = 1.000): (0) palmate / (1) not palmate.

95.* Seta 3-VI, placement $(\mathrm{CI}=0.500)$ : $(0)$ arising mesal to seta 1-VI / (1) lateral to seta 1-VI.

96. Anterior dorsomedial sclerotized plate of abdominal segment VII (CI = 0.143): (0) well developed, broad / (1) moderately developed, narrow.

97.* Anterior median process of anterior spiracular lobe (stigmal filament), development $(\mathrm{CI}=0.500)$ : $(0)$ knoblike / (1) thumb-like / (2) filamentous.

A conspicuous process in the anterior spiracular lobe is observed in An. maculatus, An. bancroftii, and An. umbrosus. In An. pollicaris occurs condition (2).

98. * Dorsal margin of long pecten teeth $(\mathrm{CI}=$ 0.333): (0) with spicules / (1) without spicules.

99 . Ventral margin of long pecten teeth $(\mathrm{CI}=0.250)$ : (0) with spicules / (1) without spicules.

100. * Pecten $(\mathrm{CI}=1.000)$ : $(0)$ with long and short teeth / (1) with long teeth only.

101. Seta $1-X(C I=0.333):(0)$ arising outside saddle / (1) inside saddle.

\section{RESULTS AND DISCUSSION}

Equally weighted parsimony analyses identified a set of 6345 parsimonious trees of 309 steps $(\mathrm{CI}=0,375$; $\mathrm{RI}=0,712 ; \mathrm{HI}=0,624)$. The successive approximations weighting analyses identified three trees that are not a subset of those 6345 equally parsimonious trees. Similarly, the implied weights analysis generated three trees, but none of them in common to previous mentioned trees. The following discussion refers to the strict consensus of three topologies obtained in the successive approximations weighting analyses (Fig. 1), which was mainly compared with the strict consensus tree of three trees recovered in the implied weights analysis (Fig. 2).

The topology shown in Fig. 1 is nearly identical to the strict consensus of three trees generated in the implied weights analysis with $K=1$ except for the placement of $A n$. annulipalpis, An. pseudobarbirostris Ludlow, An. implexus, and the Arribalzagia Series (Fig. 2). Differences between the two topologies are labeled with an asterisk (*). Generally, in both topologies, Anopheles (Anopheles) appears as a monophyletic lineage, and Bironella and Sthetomyia as sister groups outside the subgenus Anopheles. An. (Lopophodomyia) gilesi is placed basal within the clade leading to [(An. kompi, Bi. hollandi), Anopheles (Anopheles)]. The representatives of the subgenera Cellia and Nyssorhynchus collapsed outside the clade formed by Lophopodomyia, Sthetomyia, Bironella, and Anopheles (Anopheles).

The monophyly of the subgenus Anopheles (Anopheles) is poorly supported ( $\leq 50 \%$ bootstrap value) and no non-homoplastic synapomorphy was found to support the group. Members of the Laticorn Section included in the study were recovered within a monophyletic group in the implied weights analysis (Fig. 2), suggesting that the Laticorn trumpet is a derived condition for Anopheles (Anopheles), with homoplastic events within the subgenus Nyssorhynchus (An. darlingi) and Chagasia. In contrast, in the successive approximations weighting analyses, the group was recovered as a paraphyletic lineage relative to the Cycloleppteron Series. The Angusticorn Section was corroborated to be an artificial assemblage. Our results partially disagree with that of Sallum et al. (2000), which showed that neither Angusticorn nor Laticorn Sections of Anopheles (Anopheles) are natural groups.

Most relationships among members of Anopheles (Anopheles) are either moderately or poorly supported by both Bremer and bootstrap support (Fig. 1). However, as a general picture of the subgenus, it plausible to consider some better supported phylogenetic relationships, as follow. Within the Anopheles (Anopheles) only Lophoscelomyia and Arribalzagia were recovered as strongly supported monophyletic assemblages, 100\% bootstrap value and $98 \%$ bootstrap proportion, respectively (Figs 1, 2). Accordingly, Wilkerson and Peyton (1990) and Sallum et al. (2000, 2002) hypothesized the monophyly of the Arribalzagia Series. The Myzorhynchus Series is paraphyletic relative to the Cycloleppteron, Christya, and the Arribalzagia Series, while the Anopheles Series is polyphyletic with its members dispersed throughout the topology. Surprisingly, An. annulipalpis and An. grabhamii of the Cycloleppteron Series did not group together, suggesting that the series is not monophyletic.

The clade formed by An. pseudopunctipennis and An. hectoris of the Anopheles Series was recovered in an intermediary position within the subgenus Anopheles, as the sister group of the clade that includes the Laticorn Section and species of the Anopheles Series. Both Neotropical species, e. g. An. pseudopunctipennis and An. hectoris are members of a cryptic species complex. The monophyly of the Pseudopunctipennis Group was not confirmed in the present study because An. eiseni did not cluster in the clade formed by An. pseudo-punctipennis, An. hectoris. Phylogenetic relationship between An. eiseni and An. lindesayi is poorly supported $(\leq 50 \%$ bootstrap value $)$

The group consisting of An. punctipennis, An. bradleyi, and An. crucians of the Punctipennis Group and the clade formed by An. freeborni and An. atropos of the Maculipennis Group of the New World are monophyletic assemblage, placed in a basal position within the group leading to species of the Cycloleppteron, Myzorhynchus, Arribalzagia, and Christya Series (Figs 1,2). The Hyrcanus Group (represented in the study by An. hyrcanus, An. paraliae, An. nigerrimus, and An. sinensis) was recovered as a monophyletic group (57\% bootstrap value), which is sister of An. coustani (supported by $79 \%$ bootstrap value), suggesting an evolutionary relationship between the Hyrcanus and Coustani Groups as it was proposed by Reid and Knight (1961), Harrison and Scanlon (1975), and Sallum et al. (2000). Additionally, the Umbrosus Group, represented by $A n$. 
umbrosus, An. baezai and An. letifer was recovered as a moderately supported lineage ( $85 \%$ bootstrap value), similarly the monophyly of the Albotaeniatus Group ( $A n$. albotaeniatus, An. montanus) is supported by $88 \%$ bootstrap proportion.

Additionally, other monophyletic groups were recovered within Anopheles (Anopheles). For example, the clade consisting of part of the species of the Anopheles Series and the Lophoscelomyia Series includes species, which are known as "tree hole" species (An. eiseni, An. algeriensis, An. culiciformis, An. sintonoides, An. judithae, An. barberi, An. asiaticus, and An. interruptus) and "rock hole" species, e. g. An. alongensis, An. aitkenii, An. insulaeflorum, and An. lindesayi. This clade is supported by two synapomorphies: setae of dorsal claspette disposed into two apical groups and seta 3-C short, around 0.5 length of 2-C. The condition tree-hole / rock-hole probably represents a derived condition into the subgenus Anopheles.

Members of the Aitkenii, Plumbeus, and Culiciformis Groups were recovered as moderately to strongly supported monophyletic groups. An. algeriensis clustered within a monophyletic group that includes species of the Aikenii Group. Similarly, An. alongensis and members of the Culiciformis Group were recovered as a monophyletic group, corroborating the hypothesis of Reid and Knight (1961). The clade (An. alongensis plus Culiciformis Group) is supported by $88 \%$ bootstrap proportion and two synapomorphies, scutum profile short in lateral view and gonocoxite without scales.

Krzywinsky and Besansky (2003) hypothesized that there was a rapid radiation of Bironella and basal clades within Anopheles. Consequently, recovering basal relationships within the Bironella plus Anopheles lineage will be elusive, although phylogenetic analyses of combined sequence data from multiple genes seem to be promising. Based on independent lines of evidence, Chagasia was the first lineage to radiate within Anophelinae (Harbach \& Kitching 1998). Contradicting the results of the present phylogenetic analyses, sequence data of the nuclear white gene suggest that Bironella is sister to the Anopheles lineage (Krzywinsky et al. 2001). Within Anopheles, Stethomyia occupies an outside position relative to two major groups. One group formed by Cellia and Anopheles subgenera and a second group leading to the lineage ((Kerteszia, Nyssorhynchus), Lophopodomyia) (Krzywinsky \& Besansky 2003). Similarly, Sallum et al. (2002) found the same Neotropics phylogenetic grouping when using COI, COII, $18 \mathrm{~S}$ and 28 S nucleotide sequences. The subgenera Anopheles and Cellia are the most diverse and geographically dispersed. Anopheles is cosmopolitan, while Cellia occurs in the Old World.

Diversification of Anopheles (Anopheles) may have experienced rapid diversification. Consequently, recovering phylogenetic history of the group may be difficult to achieve. Results of the present phylogenetic analyses corroborated the monophyly of several groups within Anopheles (Anopheles). However, it failed to find nonhomoplastic synapomorphies to support the groups (Table III), and bootstrap and Bremer support for the split leading to distinct groups are generally poor (Figs 1,2). As a general conclusion, results of the analysis provide a phylogenetic hypothesis for the classification of Anopheles (Anopheles), and establish relationships among series and major informal groups of the subgenus. The monophyly of the subgenus Anopheles is poorly supported ( $\leq 50 \%$ bootstrap value), and none sinapomorphy was found for the group. Anopheles gilesi arises in an intermediate position between the outgroups and the clade formed by [(An. kompi, Bi. hollandi), Anopheles (Anopheles)]. Although Bironella and Sthetomyia position within Anopheles (Anopheles) clade, the support is weak and the clade aroused within a polytomy (Figs 1,2).

The Angusticorn Section seems to be an artificial group, whereas the Laticorn Section was recovered as a monophyletic group in implied weights analysis. Except for the monophyly of the Arribalzagia and Lophoscelomyia Series, which are strongly supported, no other series of the subgenus Anopheles was recovered as a monophyletic group. Monophyly of the Arribalzagia and Lophoscelomyia Series is in agreement with the hypothesis of Sallum et al. $(2000,2002)$ and Harbach and Kitching (2005). The Anopheles, Cycloleppteron, and Myzorhynchus Series were demonstrated to be polyphyletic assemblages within the subgenus Anopheles, whereas the Aitkenii, Culiciformis and Plumbeus Groups are monophyletic and include the phytotelmata species of Anopheles (Anopheles). The "tree-hole" and "rockhole" conditions seems to be derived to the subgenus Anopheles.

Contrasting to the hypothesis of Harbach and Kitching (2005), the monophyly of the Cycloleppteron Series is not supported by the results of the present phylogenetic analyses. Members of the Cycloleppteron, Myzorhynchus, Christya, and Arribalzagia Series are included in a clade which is supported by a single synapomorphy, a laticorn pupal trumpet. The Myzorhynchus Series seems to be a paraphyletic assemblage, as currently defined, because it excludes species of the Arribalzagia Series, An. implexus of the Christya Series and An. annulipalpis of the Cycloleppteron Series.

Harbach et al. (2005) described the subgenus Baimaia to include a crabhole-breeding Anopheles in Southeastern Asia, An. kyondawensis Abraham, which was originally included within the Culiciformis Group (Reid \& Knight 1961) of the subgenus Anopheles. Interestingly, in the current study the Culiciformis Group was demonstrated to be a monophyletic lineage, however the phylogenetic position of An. kyondawensis was not investigated. Harbach et al. (2005) found $A n$. kyondawensis placed as sister to Bironella plus all other Anopheles, with Chagasia as sister to these three taxa. This arrangement of taxa is in agreement with Sallum et al. (2000) hypothesis that Bironella may afford subgenus status within the genus Anopheles. Although finding strong evidence to support that both Bironella and An. kyondawensis represent independent lineages relative to the rest of Anophelinae (Harbach \& Kitching 2005), these authors did not make any classification proposal relative to Bironella, however using the principle of 
TABLE III

Character states optimization using one tree among 6345 equally parsimonious trees generated in the unweighted, unordered analysis. $\mathrm{L}=309, \mathrm{CI}=0.3754, \mathrm{RI}=0.7124^{a}$

\begin{tabular}{cr}
\hline Node & Character \\
\hline 45 & 33 \\
& 76 \\
& 98 \\
49 & 10 \\
& 30 \\
& 65 \\
& 75 \\
50 & 4 \\
& 9 \\
& 37 \\
56 & 14 \\
& 24 \\
& 27 \\
& 66 \\
& 70 \\
& 101
\end{tabular}

61

57

62

64

90

94

95

98

101

15

19

23
70

49

29

30

36

37

60

65

\section{7}

76

68

26

$69 \quad 37$

46

46

72
77

78

82

89

71

72

73

Steps Change

$1 \Rightarrow 0$

$0 \Rightarrow 1$

$0 \Rightarrow 1$

$0 \Rightarrow 1$

$1 \Rightarrow 0$

$0 \Rightarrow 1$

$0 \Rightarrow 2$

$0 \Rightarrow 1$

$0 \Rightarrow 1$

$1 \Rightarrow 2$

$1 \Rightarrow 0$

$1 \Rightarrow 0$

$0 \Rightarrow 1$

$1 \Rightarrow 0$

$0 \Rightarrow 1$

$1 \Rightarrow 0$

$1 \Rightarrow 0$

$0 \Rightarrow 1$

$0 \Rightarrow 1$

$0 \Rightarrow 1$

$0 \Rightarrow 1$

$1 \Rightarrow 0$

$1 \Rightarrow 0$

$0 \Rightarrow 1$

$0 \Rightarrow 1$

$0 \Rightarrow 1$

$0 \Rightarrow 1$

$0 \Rightarrow 3$

$0 \Rightarrow 1$

$1 \Rightarrow 0$

$0 \Rightarrow 1$

$0 \Rightarrow 1$

$1 \Rightarrow 0$

$1 \Rightarrow 0$

$1 \Rightarrow 0$

$0 \Rightarrow 1$

$0 \Rightarrow 2$

$0 \Rightarrow 2$

$0 \Rightarrow 1$

$1 \Rightarrow 0$

$0 \Rightarrow 1$

$0 \Rightarrow 1$

$1 \Rightarrow 0$

$0 \Rightarrow 1$

$0 \Rightarrow 1$

$1 \Rightarrow 0$

$1 \Rightarrow 2$

$1 \Rightarrow 0$

$0 \Rightarrow 1$

$0 \Rightarrow 1$

$1 \Rightarrow 0$

$0 \Rightarrow 2$
$1 \Rightarrow 0$

$0 \Rightarrow 1$

$1 \Rightarrow 0$

\begin{tabular}{|c|c|c|c|}
\hline Node & Character no. & Steps & Change \\
\hline & 78 & 1 & $0 \Rightarrow 1$ \\
\hline 74 & $\begin{array}{c}3 \\
32 \\
56 \\
69 \\
73 \\
75 \\
83 \\
84 \\
87\end{array}$ & $\begin{array}{l}1 \\
1 \\
1 \\
1 \\
1 \\
1 \\
1 \\
1 \\
1\end{array}$ & $\begin{array}{l}1 \Rightarrow 0 \\
0 \Rightarrow 1 \\
0 \Rightarrow 1 \\
0 \Rightarrow 1 \\
1 \Rightarrow 2 \\
0 \Rightarrow 1 \\
0 \Rightarrow 1 \\
1 \Rightarrow 0 \\
1 \Rightarrow 0\end{array}$ \\
\hline 75 & $\begin{array}{c}6 \\
9 \\
12 \\
14 \\
19 \\
22 \\
26 \\
29 \\
46 \\
72 \\
80 \\
81\end{array}$ & $\begin{array}{l}1 \\
1 \\
1 \\
1 \\
1 \\
1 \\
1 \\
1 \\
1 \\
1 \\
1 \\
1\end{array}$ & $\begin{array}{l}1 \Rightarrow 0 \\
1 \Rightarrow 0 \\
1 \Rightarrow 0 \\
1 \Rightarrow 0 \\
1 \Rightarrow 0 \\
0 \Rightarrow 1 \\
1 \Rightarrow 0 \\
0 \Rightarrow 1 \\
1 \Rightarrow 0 \\
0 \Rightarrow 1 \\
1 \Rightarrow 0 \\
0 \Rightarrow 1\end{array}$ \\
\hline 77 & $\begin{array}{l}18 \\
85 \\
88 \\
89\end{array}$ & $\begin{array}{l}1 \\
1 \\
1 \\
1\end{array}$ & $\begin{array}{l}1 \Rightarrow 0 \\
1 \Rightarrow 0 \\
0 \Rightarrow 1 \\
1 \Rightarrow 2\end{array}$ \\
\hline 78 & $\begin{array}{c}32 \\
73 \\
74 \\
80 \\
83 \\
84 \\
86 \\
91 \\
100\end{array}$ & $\begin{array}{l}1 \\
1 \\
1 \\
1 \\
1 \\
1 \\
1 \\
1 \\
1\end{array}$ & $\begin{array}{l}1 \Rightarrow 0 \\
0 \Rightarrow 1 \\
1 \Rightarrow 0 \\
2 \Rightarrow 1 \\
2 \Rightarrow 0 \\
0 \Rightarrow 1 \\
1 \Rightarrow 0 \\
1 \Rightarrow 0 \\
0 \Rightarrow 1\end{array}$ \\
\hline 80 & $\begin{array}{l}7 \\
35 \\
37 \\
41 \\
58 \\
92\end{array}$ & $\begin{array}{l}1 \\
1 \\
1 \\
1 \\
1 \\
1\end{array}$ & $\begin{array}{l}1 \Rightarrow 0 \\
1 \Rightarrow 0 \\
1 \Rightarrow 4 \\
0 \Rightarrow 1 \\
0 \Rightarrow 1 \\
1 \Rightarrow 0\end{array}$ \\
\hline 82 & $\begin{array}{c}7 \\
12 \\
28 \\
29 \\
31 \\
42 \\
46 \\
47 \\
76 \\
92\end{array}$ & $\begin{array}{l}1 \\
1 \\
1 \\
1 \\
1 \\
1 \\
1 \\
1 \\
1 \\
1\end{array}$ & $\begin{array}{l}0 \Rightarrow 1 \\
0 \Rightarrow 1 \\
0 \Rightarrow 1 \\
1 \Rightarrow 0 \\
0 \Rightarrow 1 \\
0 \Rightarrow 1 \\
0 \Rightarrow 1 \\
0 \Rightarrow 1 \\
1 \Rightarrow 0 \\
0 \Rightarrow 1\end{array}$ \\
\hline 83 & $\begin{array}{c}9 \\
19 \\
20 \\
25 \\
52 \\
67 \\
68 \\
78\end{array}$ & $\begin{array}{l}1 \\
1 \\
1 \\
1 \\
1 \\
1 \\
1 \\
1\end{array}$ & $\begin{array}{l}0 \Rightarrow 1 \\
0 \Rightarrow 1 \\
1 \Rightarrow 0 \\
1 \Rightarrow 0 \\
1 \Rightarrow 0 \\
1 \Rightarrow 0 \\
0 \Rightarrow 1 \\
3 \Rightarrow 0\end{array}$ \\
\hline
\end{tabular}


equivalent rank (Hennig 1966) designated the subgenus Baimaia to include An. kyondawensis.

\section{ACKNOWLEDGMENTS}

To RC Wilkerson, Walter Reed Army Institute of Research, Washington, DC for the loan of several specimens used in this study.

\section{REFERENCES}

Bremer K 1994. Branch support and tree stability. Cladistics 10: $295-304$.

Christophers SR 1915. The male genitalia of Anopheles. Indian J Med Res 3: 371-394.

de Pinna MCC 1991. Concepts and tests of homology in the cladistic paradigm. Cladistics 7: 367-394.

Edwards FW 1932. Genera Insectorum. Diptera, Fam. Culicidae, Fascicle 194, Desmet-Verteneuil, Bruxelles.

Farris JS 1969. A successive approximations approach to character weighting. Syst Zool 18: 374-385.

Farris JS 1982. Outgroups and parsimony. Syst Zool 31: 328-334.

Farris JS 1989. The retention index and a rescaled consistency index. Cladistics 5: 417-419.

Felsenstein J 1985. Confidence limits on phylogenies: an approach using the bootstrap. Evolution 39: 783-791.

Foley DH, Bryan JH, Yeats D, Saul A 1998. Evolution and systematics of Anopheles: insights from a molecular phylogeny of Australasian mosquitoes. Mol Phyl Evol 9: 262-275.

Harbach RE 2004. The classification of genus Anopheles (Diptera: Culicidae): a working hypothesis of phylogenetic relationships. Bull Entomol Res 94: 537-553.

Harbach RE, Kitching LJ 1998. Phylogeny and classification of the Culicidae (Diptera). Syst Entomol 23: 327-370.

Harbach RE, Kitching LJ 2005. Reconsideration of anopheline mosquito phylogeny (Diptera: Culicidae: Anophelinae) based on morphological data. Syst Biodiversity 3: 345-374.

Harbach RE, Rattanarithikul R, Harrison BA 2005. Baimaia, a new subgenus for Anopheles kyondawensis Abraham, a unique crabhole-breeding anopheline in Southeastern Asia. Proc Entomol Wash 107: 750-761.

Harrison BA 1972. A new interpretation of affinities within the Anopheles hyrcanus complex in Southeast Asia. Mosq Syst 4: 73-83.

Harrison BA, Scanlon JE 1975. Medical entomology. Studies-II. The subgenus Anopheles in Thailand (Diptera: Culicidae). Contrib Amer Entomol Inst (Ann Arbor) 12: 1-307.

Hennig W 1966. Phylogenetic Systematics, University Illinois Press, Urbana, 263 pp.

Judd DD 1996. Review of the systematics and phylogenetic relationships of the Sabethini (Diptera: Culicidae). Syst Entomol 21: 129-150.
Knight KL, Stone A 1977. A Catalog of the Mosquitoes of the World, The Thomas Say Foundation, Baltimore.

Krzywinski J, Besansky NJ 2003. Molecular systematics of Anopheles: from subgenera to subpopulations. Ann Rev Entomol 48: 111-139.

Krzywinski J, Wilkerson RC, Besansky NJ 2001a. Evolution of mitochondrial and ribosomal gene sequences in Anophelinae (Diptera, Culicidae): Implications for phylogeny reconstruction. Mol Phyl Evol 18: 479-487.

Krzywinski J, Wilkerson RC, Besansky NJ 2001b. Toward understanding Anophelinae (Diptera, Culicidae) phylogeny: insights from nuclear single copy genes and the weight of evidence. Syst Biol 50: 540-556.

Maddison WP, Maddison DR 2000. MacClade: analysis of phylogeny and character evolution. Version 4.0, Sinauer, Sunderland, MA.

Nelson G 1994. Homology and Systematics. In Brian K Hall Homology: the Hierarchical Basis of Comparative Biology, Academic Press, California.

Nixon KC, Carpenter JM 1993. On outgroups. Cladistics 9: 413-426.

Phan VT, Manh ND, Hinh TD, Vien NT 1991. Anopheles (Anopheles) cucphuongensis: a new species from Vietnam (Diptera: Culicidae). Mosq Syst 22 (1990): 145-148.

Reid JA 1968. Anopheles mosquitoes of Malaya and Borneo. Stud Inst Med Res Malaysia 31: 1-520.

Reid JA, Knight KL 1961. Classification within the subgenus Anopheles (Diptera: Culicidae). Ann Trop Med Parasitol 55: 474-488.

Sallum MAM, Peyton EL, Wilkerson RC 2005. Six new species of the Anopheles leucosphyrus group, reinterpretation of An. elegans and vector implications. Med Vet Entomol 19: 158-99.

Sallum MAM, Schultz TR, Wilkerson RC 2000. Phylogeny of Anophelinae (Diptera: Culicidae) based on morphological characters. Ann Entomol Soc Amer 93: 745-775.

Sallum MAM, Schultz TR, Foster PG, Wirtz RA, Wilkerson RC 2002. Phylogeny of Anophelinae (Diptera: Culicidae) based on nuclear ribosomal and mitochondrial DNA sequences. Syst Entomol 27: 361-381.

Sorenson MD 1999. TreeRot, v. 2.1, Boston University, Boston.

Swofford DL 2003. PAUP*. Phylogenetic analysis using parsimony (*and other methods), version 4.0b10. Sinauer, Sunderland, MA.

Wilkerson RC, Peyton EL 1990. Standardized nomenclature for the costal wing spots of the genus Anopheles and other spotted-winged mosquitoes (Diptera: Culicidae). J Med Entomol 27: 207-224.

Zavortink TJ 1969. Mosquito Studies (Diptera, Culicidae). XV. Anew species of treehole breeding Anopheles from the southeastern United States. Contrib Amer Entomol Inst (Ann Arbor) 4: 27-34. 
